\title{
Fictitious Students Creation Incentives in School Choice Problems
}

\author{
Mustafa Oğuz Afacan*
}

August 27, 2013

Faculty of Arts and Social Sciences, Sabancı University, 34956, İstanbul, Turkey.

\begin{abstract}
We identify a new channel through which schools can potentially manipulate the wellknown student and school optimal stable mechanisms. We introduce two different fictitious students creation manipulation notions where one of them is stronger. While the student and school optimal stable mechanisms turn out to be weakly fictitious student-proof under acyclic (Ergin (2002)) and essentially homogeneous (Kojima (2011a)) priority structures, respectively, they still lack strong fictitious student-proofness. We, then, compare the mechanisms in terms of their vulnerability to manipulations in the sense of Pathak and Sönmez (2013) and find out that the student-optimal stable mechanism is more manipulable than the school-optimal one. Lastly, in the large market setting of Kojima and Pathak (2009), the student-optimal stable mechanism becomes weakly fictitious student-proof as the market is getting large.
\end{abstract}

JEL classification: C71, C78, D71, D78, J44.

Keywords: the student-optimal stable mechanism, the school-optimal stable mechanism, fictitious students, acyclicity, essential homogeneity, large market.

${ }^{*}$ E-mail: moguzafacan@gmail.com 


\section{Introduction}

Initiated by Gale and Shapley (1962), matching theory has been fruitful both in theory and practice. Theoretical findings have been successfully applied to real-life problems including doctor assignments, student placements, and kidney exchanges. It has been documented that the theoretically appealing stability notion of Gale and Shapley (1962) has also proved to be very critical for the well-working of real-life matching markets. ${ }^{1}$

Fortunately, Gale and Shapley (1962) show the existence of a stable solution through introducing the celebrated deferred acceptance algorithm. This positive result, however, has not solved all the problems of matching market design, especially regarding the strategic ones. Roth (1982) shows that no stable mechanism is immune to preference manipulations. However, in the one-to-one matching setting, whenever either side's preferences are common knowledge, then there exists a stable and strategy-proof mechanism (Dubins and Freedman (1981), Roth (1982)). As well as preference manipulations, Sönmez (1997, 1999) show that no stable mechanism is immune to capacity and pre-arrangement manipulations, respectively. Some recent related papers on manipulation incentives in matching markets include Kojima (2011b), Kojima and Pathak (2009), Afacan (2012, 2011), and Kesten (2012).

In the current study, we investigate another channel via which schools could potentially manipulate matching mechanisms. Yokoo et al. (2004) study false-bid manipulation incentives in combinatorial auctions. In this kind of manipulations, a bidder tries to profit from submitting false bids under a fictitious name. On the other hand, as a corresponding manipulation in the school choice setting, one can think of a situation where schools create fictitious students in the hope of getting better assignments. This raises the question of whether schools can manipulate matching mechanisms via creating fictitious students and we address this issue in the current paper.

In order to model fictitious student creation incentives, we first introduce two different manipulation notions where one of them is stronger. In the strong one, schools encounter

\footnotetext{
${ }^{1}$ For an excellent source, refer to Roth and Sotomayor (1990).
} 
two natural constraints in creating fictitious students: They can not affect the preference profile of "non-fictitious" (real) students and the relative priority rankings of schools over them. On the other hand, for the weak one, we also impose that fictitious students have to be either unassigned or matched with the school which created them. Then, unfortunately, it turns out that both the student and school-optimal stable mechanisms are not even weakly fictitious student-proof.

Given the above negative result, we look for some structure on the primitives helping us to gain fictitious student-proofness. The extant literature shows that the student-optimal stable mechanism admits many good properties (including strategic ones) under Ergin (2002)'s acyclic priority structures, ${ }^{2}$ which makes acyclicity a worthwhile condition to consider. It turns out that the student-optimal stable mechanism becomes weakly fictitious student proof under acyclicity, however, it still lacks strong fictitious student-proofness. On the other hand, the school-optimal stable mechanism is not weakly fictitious student-proof even under acyclicity. This leads us to look for a stronger condition for the school-optimal stable rule. A recent paper Kojima (2011a) shows that, in the many-to-many matching environment, the student-optimal stable mechanism becomes strategy-proof and Pareto efficient if and only if the priority structure is "essentially homogeneous". Fortunately, essential homogeneity proves useful in the current paper as well: The school-optimal stable mechanism is weakly fictitious student-proof if schools' priorities are essentially homogeneous.

In spite of the above positive results, both acyclicity and essential homogeneity require strong conditions in that the schools' priorities would barely satisfy them. Given this fact, instead of assuming them, we compare the manipulability of the mechanisms on problem basis as done by Pathak and Sönmez (2013). It turns out that the student-optimal stable mechanism is more manipulable than the school-optimal one in the sense that the former is strongly manipulable via creating fictitious students at any problem where so is the latter, and there is a problem instance at which the latter is not manipulable, yet, the former is.

\footnotetext{
${ }^{2}$ Please refer to Related Literature section for detailed references illustrating this point.
} 
Lastly, we investigate the scope of fictitious student manipulations under the studentoptimal stable mechanism in large markets. The existing literature (Roth and Peranson (1999), Immorlica and Mahdian (2005), Kojima and Pathak (2009), and Hatfield et al. (2011)) shows that some of undesirable properties of mechanisms may disappear as the number of participants goes to infinity. Motivated by this fact, we employ the large market setting of Kojima and Pathak (2009) and address the question of whether the same is true for fictitious student manipulations. To this end, we show that instead of considering fictitious student manipulations, we can consider a certain type of priority misreporting in the sense that whenever there is a room for manipulation of the former kind, then so is there of the latter kind. This result enables us to directly apply the results of Kojima and Pathak (2009) for fictitious student manipulations as well: The student-optimal stable mechanism becomes weakly fictitious student-proof under some regularity conditions as the number of participants goes to infinity.

Why should we care about fictitious students creation manipulations? From the wellknown comparative statistics result of Gale and Sotomayor (1985), we know that, under both the student and school optimal stable mechanisms, the presence of fictitious students leads real ones to be at least weakly worse off, and in the case of a successful manipulation, at least one of them is strictly worse off. This means that manipulation results in Pareto inferior outcomes to the ones that would otherwise arise. ${ }^{3}$ Hence, this fact suggests the benevolent social planner to take this kind of manipulation possibilities into account in matching market design. A related policy recommendation of the paper is that the social planner might influence the schools' priorities in a way that makes them acyclic (essentially homogeneous) to avoid manipulations under the student (school)-optimal stable mechanism.

Two conceptual issues are whether schools are strategic agents and whether their priorities reflect their actual preferences. In the conventional school choice model, it is assumed that schools are just objects to be consumed and their priorities are exogenously given based

\footnotetext{
${ }^{3}$ Since schools are considered as objects to be consumed in the school choice problems, only the welfare of students matters (in our setting, naturally, only the welfare of real ones is considered).
} 
on certain criteria imposed by law. ${ }^{4}$ Hence, neither they are assumed to be strategic agents nor their priorities necessarily reflect their actual preferences. However, there are some student placement systems where schools can influence their priorities implying that they can be strategic and their priorities do indeed reflect their actual preferences. The New York City and Boston schools districts (see Abdulkadiroglu (2011) for details), which are the two largest student placement system in USA, are examples for such school choice problems. On the other hand, while we demonstrate our results in the school choice context, our analysis is perfectly applicable to the two-sided intern-hospital matching market setting. ${ }^{5}$ Hospitals might potentially manipulate matching mechanisms via creating fictitious doctors and, in this setting, hospitals completely form their preferences themselves, hence, they can be strategic.

Another related concern is whether schools manipulate mechanisms via creating fictitious students in real-life problems? This paper demonstrates the potential for such manipulations rather than claiming that they certainly exist in real-life problems. Indeed, generally speaking, it is very difficult to identify manipulations even the well-known ones: preference, capacity, pre-arrangements in real-life problems. Even though one figures out that a participant misreports its private information, it is difficult to argue that it does so in the hope of getting better outcome. The current paper like other similar works in the existing literature discovers a new kind of manipulation and conducts theoretical analysis.

\section{Related Literature}

This paper is broadly related to the extensive literature on manipulations in matching markets. In the two-sided matching context, Roth (1982) shows that no stable mechanism is strategy-proof. ${ }^{6}$ Nonetheless, in the one-to-one matching setting, if one side of the

\footnotetext{
${ }^{4}$ Such as the proximity of students' houses to schools and which schools their siblings are attending (if applicable).

${ }^{5}$ In US, interns and hospitals are matched through the centralized matching program NRMP each year, and intern-optimal stable mechanism is being used (Roth (1984)).

${ }^{6} \mathrm{~A}$ mechanism is strategy-proof if no agent ever benefits from misreporting his preference.
} 
market has commonly known preferences, then there exists a strategy-proof stable mechanism (Dubins and Freedman (1981),Roth (1982)). As well as preference manipulations, Sönmez $(1997,1999)$ prove that no stable mechanism is non-manipulable via capacities and pre-arrangements respectively. Similarly, Afacan (2011) shows that no stable mechanism is immune to application fee manipulations. Given these impossibility results, Pathak and Sönmez (2013) introduces a new methodology to compare mechanisms by their vulnerability to manipulations based on the room for strategizing across problems.

The acyclicity (Ergin (2002)) and essential homogeneity (Kojima (2011a)) conditions prove critical in the current paper. There are other related studies in the literature sharing the same point. Ergin (2002) shows that the student-optimal stable mechanism is group strategy-proof $^{7}$ under acyclic priority structures. Kojima (2011b) demonstrates that no individual student is better off by first misreporting his preference and then appealing to the outcome under acyclicity. This result is, then, generalized to the groups of students by Afacan (2012). Moreover, Kesten (2012) proves that acyclicity is necessary and sufficient for the student-optimal stable mechanism to be immune to capacity manipulations. In a recent study, Kojima (2011a) shows that the student-optimal stable mechanism is separately efficient and strategy-proof in the many-to-many matching setting if and only if the priority structure of schools is essentially homogeneous. The current paper identifies one more sense in which such priority structures are important for the matching market design.

While there are many negative results in finite matching markets, some of them have been shown to disappear in large markets. In the one-to-one matching setting, Immorlica and Mahdian (2005) demonstrate that schools' manipulation incentives vanish as the market is getting large. This result is, then, generalized to the many-to-one matching environment under certain conditions by Kojima and Pathak (2009). ${ }^{8}$ Moreover, in a recent paper, Hatfield et al. (2011) look at the incentives of schools to improve themselves and they show

\footnotetext{
${ }^{7} \mathrm{~A}$ mechanism is group strategy-proof if no group of agents ever has incentive to misreport their preferences.

${ }^{8}$ Kojima and Pathak (2009) also do some equilibrium analysis.
} 
that stable mechanisms give right incentives in large markets, whereas, they fail to do so in finite markets.

Another related paper from the auction theory literature is Yokoo et al. (2004) where the authors examine the incentives of bidders to submit bids under fictitious names in combinatorial auctions. They say that an auction protocol is false-name-proof if no bidder can profitably submit a false name bid in any problem instance. They first show that no efficient auction protocol is false-name-proof then give a sufficient condition which makes VCG mechanism false-name-proof. One could interpret fictitious student creation manipulation as the counterpart of false name bidding in the matching context.

\section{$3 \quad$ Model \& Results}

A school choice problem consists of a tuple $(S, C, P, \succ, q)$. The first two components are finite and disjoint sets of students and schools, respectively. Each student $i \in S$ has a preference relation $P_{i}$, which is a complete, strict, and transitive binary relation over the set of schools $C$ and being unassigned (denoted by $\emptyset$ ). Let $\mathcal{P}$ be the set of all such preference relations and the list $P=\left(P_{i}\right)_{i \in S}$ is the preference profile of students. We write $c R_{i} c^{\prime}$ if either $c P_{i} c^{\prime}$ or $c=c^{\prime}$. Each school $c \in C$ has a priority order $\succ_{c}$, which is a complete, strict, and transitive binary relation over the set of students $S$ and keeping seat vacant, denoted by $\emptyset$. We write $\succ=\left(\succ_{c}\right)_{c \in C}$ for the priority order profile of schools. The last component $q=\left(q_{c}\right)_{c \in C}$ is the quota profile of schools where $q_{c}$ is of school $c$. We call the tuple $(\succ, q)$ priority structure.

We interpret the priority orders of schools as their preferences and extend them to over the set of groups of students in the responsive (Roth (1985)) way. Then, the choice of a school $c \in C$ from a group of students $J \subseteq S$ under the priority order $\succ_{c}$ and quota $q_{c}$ is defined as

$$
C h_{c}\left(\succ_{c}, q_{c}, J\right)=\left\{J^{\prime} \subseteq J:\left|J^{\prime}\right| \leq q_{c}, J^{\prime} \succ_{c} \quad J^{\prime \prime} \text { for all } J^{\prime \prime} \subseteq J \text { such that }\left|J^{\prime \prime}\right| \leq q_{c}\right\}
$$


A matching $\mu$ is an assignment of students to schools such that no student is assigned more than one school, and no school is assigned to more students than its quota. We write $\mu_{k}$ for the assignment of student (school) $k \in S \cup C$ under $\mu$. A matching $\mu$ is individually rational if $\mu_{i} R_{i} \emptyset$ for all $i \in S$ and, for any $c \in C, C h_{c}\left(\succ_{c}, q_{c}, \mu_{c}\right)=\mu_{c}$. Matching $\mu$ is blocked by a student-school pair $(i, c) \in S \times C$ if $c P_{i} \mu_{i}$ and $i \in C h_{c}\left(\succ_{c}, q_{c}, \mu_{c} \cup\{i\}\right)$. A matching $\mu$ is stable if it is individually rational and is not blocked by any pair $(i, c) \in S \times C$.

A mechanism $\psi$ is a systematic way to assign a matching for every problem. Mechanism $\psi$ is stable if its outcome is stable at every problem instance. In the rest of the paper, as $q$ and $C$ will be fixed, we often write $\psi(S, P, \succ)$ for the outcome and, for ease of notation, we just write $\psi(P)$ whenever it does not cause confusion.

Below, we outline the student-proposing deferred acceptance algorithm (Gale and Shapley (1962)) producing the student-optimal stable matching.

Step 1. Each student applies to his first choice school. Each school that receives one or more offers holds as many best acceptable offers as at most its quota and rejects the rest.

In general,

Step t. Each student who was rejected in step $(t-1)$ applies to his best acceptable choice in the set of schools to which he did not apply before. Each school holds as many best acceptable offers as at most its quota among the set of offers held at step $(t-1)$ and the offers it receives at this step and rejects the rest.

The algorithm terminates when no student applies to a school, and the tentatively held offers at the termination step are realized as assignments. The student-optimal stable mechanism produces the student-optimal stable matching for every problem. On the other hand, the school-proposing version of the above algorithm produces the school-optimal stable matching. Similarly, the school-optimal stable mechanism assigns the school-optimal stable matching for every problem. In the rest of the paper, we write $\psi^{S}$ and $\psi^{C}$ for the student 
and school-optimal stable mechanism, respectively.

Definition 1. Mechanism $\psi$ is weakly manipulable via creating fictitious students at a matching problem instance $(S, C, P, \succ, q)$ if there exist a school $c \in C$ and another matching problem instance $\left(S^{\prime}, C, P^{\prime}, \succ^{\prime}, q\right)$ such that the followings satisfy:

(i) $S \subset S^{\prime}$,

(ii) for all $i, j \in S$ and $c \in C, i \succ_{c} j$ if and only if $i \succ_{c}^{\prime} j$,

(iii) $P_{i}^{\prime}=P_{i}$ for all $i \in S$,

(iv) $\psi_{c}\left(S^{\prime}, P^{\prime}, \succ^{\prime}\right) \cap S \succ_{c} \psi_{c}(S, P, \succ)$.

In words, we refer to the students in $S^{\prime} \backslash S$ as fictitious students and say that a mechanism is weakly manipulable via creating fictitious students at a problem if a school can be strictly better off by creating such students under the constraints that it can affect neither the priority rankings of schools among non-fictitious students (Condition $(i i)$ ) nor the preference profile of them (Condition $(i i i)$ ).

Remark 1. In the definition, manipulating school $c$ compares the outcomes based on its non-fictitious students assignments (i.e, according to $\succ_{c}$ rather than $\succ_{c}^{\prime}$ ). This is very natural since it knows that all students in the set $S^{\prime} \backslash S$ are fictitious created by itself.

Definition 2. A mechanism $\psi$ is strongly fictitious student-proof if it is not weakly manipulable via creating fictitious students at any matching problem instance $(S, C, P, \succ, q)$.

Proposition 1. Neither $\psi^{S}$ nor $\psi^{C}$ is strongly fictitious student-proof.

Proof. Consider a problem consisting of $S=\{i\}$ and $C=\{a, b\}$ with $q_{a}=q_{b}=1$. Assume that student $i$ prefers school $a$ to school $b$ to being unassigned, that is, $P_{i}: a, b, \emptyset$. The priorities of schools are such that $\succ_{a}=\succ_{b}: i, \emptyset$. Then, $\psi_{i}^{C}\left(P_{i}\right)=\psi_{i}^{S}\left(P_{i}\right)=a$.

Now, let school $b$ create fictitious student $j$ with $P_{j}: a, \emptyset$. For the priority order of schools over $\{i, j\}$, assume that $\succ_{a}^{\prime}=\succ_{b}^{\prime}: j, i, \emptyset$. Then, $\psi_{i}^{C}\left(P_{i}, P_{j}\right)=\psi_{i}^{S}\left(P_{i}, P_{j}\right)=b$. Hence, school $b$ is better off via creating fictitious student $b$, which finishes the proof. 
The above negative result is indeed very well expected as it is easy to see that whenever a school does not match with its top priority group under any stable rule, then it can manipulate the mechanism. Hence, in what follows, we weaken the manipulation concept and investigate whether the mechanisms are manipulable via creating fictitious students in this weak sense.

Definition 3. Mechanism $\psi$ is strongly manipulable via creating fictitious students at a matching problem instance $(S, C, P, \succ, q)$ if there exist a school $c \in C$ and another matching problem instance $\left(S^{\prime}, C, P^{\prime}, \succ^{\prime}, q\right)$ such that the followings satisfy:

(i) $S \subset S^{\prime}$,

(ii) for all $i, j \in S$ and $c \in C, i \succ_{c} j$ if and only if $i \succ_{c}^{\prime} j$,

(iii) $P_{i}^{\prime}=P_{i}$ for all $i \in S$,

(iv) for all $i \in S^{\prime} \backslash S$, either $\psi_{i}\left(S^{\prime}, P^{\prime}, \succ^{\prime}\right)=$ c or $\psi_{i}\left(S^{\prime}, P^{\prime}, \succ^{\prime}\right)=\emptyset$,

(v) $\psi_{c}\left(S^{\prime}, P^{\prime}, \succ^{\prime}\right) \cap S \succ_{c} \psi_{c}(S, P, \succ)$.

The only difference between the two manipulation concepts is Condition (iv) in the above definition. Namely, it imposes the restriction that fictitious students have to be either unassigned or assigned the school which created them. This condition, which we interpret as capturing the situations where it is not in the schools' interest to create such students where some of them get matched with a school other the manipulating one, can realize in real-life matching markets in the presence of some policy ${ }^{9}$ or externalities ${ }^{10}$ (even though we do not consider externalities in the paper).

\footnotetext{
${ }^{9}$ One such policy might impose high penalties whenever a school reports that one of its assigned students withdrew from his seat on all schools except the reporting one.

${ }^{10}$ One kind of such externalities might correspond to the case where each school prefers other schools to be matched with their better options. Under the weak manipulation case (Definition 1), a manipulating school might cause all other schools to be strictly worse-off (see the example given in the proof of Proposition 1: since student $j$ is fictitious there, at the end, school $a$ would end up with no student which is strictly worse outcome than what would otherwise arise). Whereas, by the well-known comparative statistics result (Gale and Sotomayor (1985)), whenever a school manipulates the student-optimal stable mechanism through creating fictitious students in the strong sense (Definition 3), then all schools would be at least weakly better off, with at least one of them being strictly better off as well as the manipulating one.
} 
Definition 4. A mechanism $\psi$ is weakly fictitious student-proof if it is not strongly manipulable via creating fictitious students at any matching problem instance $(S, C, P, \succ, q)$.

Proposition 2. Neither $\psi^{S}$ nor $\psi^{C}$ is weakly fictitious student-proof.

Proof. We first prove the manipulability of $\psi^{S}$. Consider a matching problem instance consisting of $S=\{i, j\}, C=\{a, b\}, q_{a}=q_{b}=1$, the following preference and priority order profiles:

$$
\begin{aligned}
& P_{i}: b, a, \emptyset, \\
& P_{j}: a, b, \emptyset, \\
& \succ_{a}: i, j, \emptyset, \\
& \succ_{b}: j, i, \emptyset .
\end{aligned}
$$

Let $P=\left(P_{i}, P_{j}\right)$, then $\psi^{S}(P)=\left(\psi_{i}^{S}(P), \psi_{j}^{S}(P)\right)=(b, a)$. Now, let school $b$ create a fictitious student $k$ and assume that the preference $P_{k}$ and the priority rankings of schools $\succ^{\prime}$ over $\{i, j, k\}$ are as follows:

$$
\begin{gathered}
P_{k}: a, \emptyset, b, \\
\succ_{a}^{\prime}: i, k, j, \emptyset, \\
\succ_{b}^{\prime}: j, i, k, \emptyset .
\end{gathered}
$$

Let $P^{\prime}=\left(P_{i}, P_{j}, P_{k}\right)$. Then, $\psi^{S}\left(P^{\prime}\right)=\left(\psi_{i}^{S}\left(P^{\prime}\right), \psi_{j}^{S}\left(P^{\prime}\right), \psi_{k}^{S}\left(P^{\prime}\right)\right)=(a, b, \emptyset)$ (note that all the conditions in the manipulation definition are met). Hence, school $b$ is better off through creating fictitious student $k$.

For the manipulability of $\psi^{C}$, let us consider the same problem as above with the difference that $q_{a}=2$. Then, $\psi^{C}(P)=\left(\psi_{i}^{C}(P), \psi_{j}^{C}(P)\right)=(b, a)$. Let school $a$ create fictitious student $k$ with the same preference profile $P^{\prime}$ and same priorities $\succ^{\prime}$ as above. Then, $\psi^{C}\left(P^{\prime}\right)=\left(\psi_{i}^{C}\left(P^{\prime}\right), \psi_{j}^{C}\left(P^{\prime}\right), \psi_{k}^{C}\left(P^{\prime}\right)\right)=(a, b, a)$, making school $a$ better off.

Given the lack of even weak fictitious student-proofness, we look for some condition on the primitives helping us to recover at least some of the above negative results. The 
extant literature shows that Ergin (2002)'s acyclicity condition has been very useful in that sense. Specifically, the student-optimal stable mechanism admits many good properties including strategic ones under acyclicity condition. In what follows, we therefore investigate the fictitious student creation incentives under that condition.

Definition 5 (Ergin (2002)). Given a priority structure $(\succ, q)$, a cycle is $a, b \in C, i, j, k \in S$ such that;

(i) $i \succ_{a} j \succ_{a} k$ and $k \succ_{b} i$, and

(ii) there exist (possibly empty) disjoint sets of students $S_{a}, S_{b} \subseteq S \backslash\{i, j, k\}$ such that $\left|S_{a}\right|=q_{a}-1,\left|S_{b}\right|=q_{b}-1, s \succ_{a} j$ for every $s \in S_{a}$, and $s \succ_{b} i$ for every $s \in S_{b}$.

A priority structure $(\succ, q)$ is acyclic if there exists no cycle.

Definition 6. Mechanism $\psi$ is weakly (strongly) manipulable via creating fictitious students under acyclicity at a matching problem instance $(S, C, P, \succ, q)$ if there exist a school $c \in C$ and another matching problem instance $\left(S^{\prime}, C, P^{\prime}, \succ^{\prime}, q\right)$ such that $(i)$ all the conditions in Definition 1 (Definition 3) are met, and $(i i)\left(\succ^{\prime}, q\right)$ is acyclic.

Definition 7. A mechanism $\psi$ is strongly (weakly) fictitious student-proof under acyclicity if it is not weakly (strongly) manipulable via creating fictitious students under acyclicity at any matching problem instance $(S, C, P, \succ, q)$.

Unfortunately, given that the priority structure $\left(\succ^{\prime}, q\right)$ in the proof of Proposition 1 is acyclic, it turns out that both the student and school optimal stable mechanisms are weakly manipulable via creating fictitious students.

Corollary 1. Neither $\psi^{S}$ nor $\psi^{C}$ is strongly fictitious student-proof under acyclicity.

Below, we obtain the first sharp difference between the student and school optimal stable mechanisms in terms of fictitious student creation incentives under acyclic priority structures. 
Theorem 1. While $\psi^{S}$ is weakly fictitious student-proof under acyclicity, $\psi^{C}$ is not.

Proof. See Appendix.

Given that the priority structure in the proof of Proposition 2 for the manipulability of $\psi^{S}$ is not acyclic, we obtain a necessary and sufficient condition in terms of priority structures in the sense that there is a problem instance where a school can succeed in manipulation through creating fictitious students in the absence of the acyclicity imposition, whereas, it is otherwise impossible as the above theorem shows.

$\psi^{C}$ being manipulable even under acyclicity leads us to look for more stringent priority structures. In a recent paper, Kojima (2011a) considers the many-to-many matching setting and shows that, as opposed to the many-to-one setting, the student-optimal stable mechanism is neither strategy-proof nor weakly Pareto efficient. Then, he introduces the so called "essentially homogeneous" priority structures, which are more stringent than acyclic ones, and proves that the student-optimal stable mechanism recovers those properties (indeed, it becomes Pareto efficient) if and only if the schools' priority structure is essentially homogeneous. In what follows, we will show that the same is true for weak strategy-proofness of $\psi^{C}$ as well.

Definition 8 (Kojima (2011a)). A priority structure $(\succ, q)$ is essentially homogeneous if there exist no $a, b \in C$ and $i, j \in S$ such that

(i) $i \succ_{a} j$ and $j \succ_{b} i$, and

(ii) There exist sets of students $S_{a}, S_{b} \subseteq S \backslash\{i, j\}$ such that $\left|S_{a}\right|=q_{a}-1,\left|S_{b}\right|=q_{b}-1$, $k \succ_{a} j$ for every $k \in S_{a}$, and $k \succ_{b} i$ for every $k \in S_{b}$.

Remark 2. It is easy to see that essentially homogeneous priority structures are acyclic, yet, the converse is not true.

Definition 9. Mechanism $\psi$ is weakly (strongly) manipulable via creating fictitious students under essential homogeneity at a matching problem instance $(S, C, P, \succ, q)$ if there exist a 
school $c \in C$ and another matching problem instance $\left(S^{\prime}, C, P^{\prime}, \succ^{\prime}, q\right)$ such that $(i)$ all the conditions in Definition 1 (Definition 3) are met, and $(i i)\left(\succ^{\prime}, q\right)$ is essentially homogeneous.

We define strong (weak) fictitious student-proofness under essential homogeneity in the same way as in Definition 7. As the priority structure $\left(\succ^{\prime}, q\right)$ in the proof of Proposition 1 is essentially homogeneous, we have the following result.

Corollary 2. Both $\psi^{S}$ and $\psi^{C}$ are not strongly fictitious student-proof under essential homogeneity.

However, we recover the weak fictitious student-proofness of $\psi^{C}$ with the help of essential homogeneity.

Theorem 2. $\psi^{C}$ is weakly fictitious student-proof under essential homogeneity.

Proof. See Appendix.

As the priority structure given in the proof of Proposition 2 for the manipulability of $\psi^{C}$ is not essentially homogeneous, it is also necessary condition in the sense that there is a problem instance where a school can succeed in manipulation through creating fictitious students in the absence of the essential homogeneity imposition, whereas, it is otherwise impossible as the above result shows.

Remark 3. In the manipulation notions, we assume that the priority rankings of fictitious students in schools can be arranged in any way (as long as it is acyclic or essentially homogeneous in the corresponding parts of the paper) by the school which created them. However, the schools' priorities might be correlated, hence, it might not be possible. ${ }^{11}$ While we need this assumption in order to make analysis possible, our results would not be affected by the absence of it. It is clear that manipulation would be harder without it, which implies

\footnotetext{
${ }^{11}$ For example, think of a situation where the manipulating school wants a fictitious student to be at the top of the priority order of school $a$ while at the bottom in that of school $b$. This, however, might not be possible if the qualifications of the fictitious student, which make him top at the priority order of school a, also put his name in a high position in that of school $b$ as well.
} 
that acyclicity (essential homogeneity) would still be sufficient for the student (school)optimal stable mechanism to be weakly fictitious student-proof. On the other hand, since all the examples for the negative results given in the paper would also work, ${ }^{12}$ the necessity of them would be still valid as well.

Theorem 1\&2 provide conditions in terms of priority structures making the mechanisms weakly fictitious student-proof. While acyclicity is less demanding than essential homogeneity, it does not necessarily mean that the school-optimal stable mechanism is more manipulable than the student-optimal stable rule in the sense that whenever the latter is manipulable at a problem instance, then so is the former. This kind of manipulability comparison between mechanisms has been done by Pathak and Sönmez (2013), and the following notion is taken from their work.

A mechanism $\psi$ is at least as manipulable as mechanism $\phi$ via creating fictitious students if whenever the latter is strongly manipulable at a problem, then so is the former at the same problem. Mechanism $\psi$ is more manipulable via creating fictitious students than $\phi$ if it is at least as manipulable as $\phi$ via creating fictitious students, and there exists a problem instance at which the former is strongly manipulable, whereas, the latter is not.

Theorem 3. $\psi^{S}$ is more manipulable via creating fictitious students than $\psi^{C}$.

Proof. See Appendix.

Remark 4. We only consider strong manipulations in the above manipulability comparison analysis. As pointed out previously, under any stable rule, if a school is not matched with its top priority group of students, then it has incentive to manipulate the mechanism in the weak sense. This basically implies that whenever the school-optimal stable mechanism is weakly manipulable, then so is the student-optimal one, and vice-versa. Hence, we can not say either one is more manipulable than the other one in terms of weak manipulations.

\footnotetext{
${ }^{12}$ Basically, in the examples, the relevant underlyings of schools might enable the manipulating schools to arrange the priority orders as in the proofs even without our assumption.
} 


\subsection{Large Market Analysis}

Large market analysis has proved to be fruitful in recovering some negative results in finite markets. Motivated by this fact, in this section, we show that the student-optimal stable mechanism becomes weakly fictitious student-proof as the market is getting large under the regularity conditions of Kojima and Pathak (2009). For the proof, we first show that whenever there is a room for strong manipulation via creating fictitious students, then so is there for a certain type of priority misreporting so called "dropping strategy". That is, this result says that we can consider priority misreporting incentives of schools rather than fictitious students creation incentives. This enables us to directly apply Kojima and Pathak (2009)' result to fictitious student creation manipulations.

A reported priority list is said to be a dropping strategy if it simply declares some students who are acceptable under the true priority list as unacceptable. Formally, a dropping strategy is a report $\succ_{c}^{\prime}$ such that $(i) s \succ_{c} s^{\prime}$ and $s \succ_{c}^{\prime} \emptyset$ imply $s \succ_{c}^{\prime} s^{\prime}$ and $(i i) \emptyset \succ_{c} s$ implies $\emptyset \succ_{c}^{\prime} s$.

Lemma 1. Given a problem instance and a stable mechanism, suppose that the mechanism is strongly manipulable by school c via creating fictitious students and matching $\mu$ is produced through the manipulation. Then, there exists a dropping strategy of school c producing a matching which is at least as good as $\mu$ for school $c$.

Proof. See Appendix.

In the rest of this section, we employ the large market setting of Kojima and Pathak (2009). For the sake completeness, we fully describe it below.

A random market is a tuple $\Gamma=\left(S, C, \succ_{c}, k, \mathcal{D}\right)$. Here, $k$ is a positive integer representing the length of students' preferences, that is, the number of acceptable schools that students can declare in their preferences. On the other hand, $\mathcal{D}=\left(p_{c}\right)_{c \in C}$ is a probability distribution over $C$. Each student $i$ 's preference unfolds as follows:

Step 1. Select a school independently from $\mathcal{D}$ and list this school as the top choice of student $i$. 
In general,

Step $\mathbf{t} \leq \mathbf{k}$. Select a school independently from $\mathcal{D}$ until a previously undrawn school is drawn. List that school as the $t^{\text {th }}$ choice of student $i$.

A sequence of random markets is denoted by $\left(\tilde{\Gamma}^{1}, \tilde{\Gamma}^{2}, \ldots\right)$ where $\tilde{\Gamma}^{n}=\left(C^{n}, S^{n}, \succ_{C^{n}}, k^{n}, \mathcal{D}^{n}\right)$ is a random market in which $\left|C^{n}\right|=n$.

Definition 10 (Kojima and Pathak (2009)). A sequence of random markets $\left(\tilde{\Gamma}^{1}, \tilde{\Gamma}^{2}, \ldots\right)$ is regular if there exist positive integer $k$ and $\bar{q}$ such that

(i) $k^{n}=k$ for all $n$,

(ii) $q_{c} \leq \bar{q}$ for $c \in C^{n}$ for all $n$,

(iii) $\left|S^{n}\right| \leq \bar{q} n$ for all $n$, and

(iv) for all $n$ and $c \in C^{n}$, any $s \in S^{n}$ is acceptable to $c$.

Given a random market $\tilde{\Gamma}^{n}$, the expected number of schools that can strongly manipulate the student-optimal stable mechanism via creating fictitious students when others are truthful (i.e., when others do not manipulate via creating fictitious students), denoted by $\alpha(n)$, is given below:

$\alpha(n)=E\left[\#\left\{c \in C^{n}:\right.\right.$ school $c$ can strongly manipulate $\psi^{S}$ via creating fictitious students in the induced problem $\left(S^{n}, C^{n}, P, \succ^{n}, q^{n}\right)$ when other schools are truthful $\left.\} \mid \tilde{\Gamma}^{n}\right]$.

Due to Lemma 1, we can directly apply the result of Kojima and Pathak (2009), hence, we have the following theorem.

Theorem 4. If the sequence of random markets is regular, then the expected proportion of schools that can strongly manipulate $\psi^{S}$ via creating fictitious students when others are truthful, $\alpha(n) / n$, converges to zero as the number of colleges goes to infinity.

Remark 5. We have the above large market result for strong manipulations. On the other hand, Lemma 1 is not true for the weak manipulation. That is, a school might not have a dropping strategy giving an outcome which is at least as good as the outcome induced 
through a weak fictitious student creation manipulation. For instance, consider a problem consisting of $S=\{i\}$ and $C=\{a, b\}$ with $q_{a}=q_{b}=1$. Student $i$ prefers school $a$ to $b$ to being unassigned and both schools prefer him to keeping the seat vacant. Then, under any stable mechanism, student $i$ is matched with school $a$, and school $b$ can not be better off by misreporting its priority. However, it can create a fictitious student $j$ with $P_{j}: a, b, \emptyset$ and $\succ_{a}^{\prime}: j, i, \emptyset$. Then, student $i$ is matched with school $b$ under any stable rule, making it better off.

\section{Conclusion \& Discussion}

We investigate the fictitious student creation incentives of schools under the student and school optimal stable rules. The former is weakly fictitious student-proof under acyclicity, and so is the latter under essential homogeneity. Even though essential homogeneity requires a more stringent condition than acyclicity, the student optimal stable mechanism turns out to be more manipulable than the school optimal stable rule. As opposed to these negative results in finite markets, the student-optimal stable rule becomes weakly fictitious studentproof as the market is getting large.

In our analysis, we assume that students might be unacceptable to schools. ${ }^{13}$ While there are some schools districts where students can be unacceptable, ${ }^{14}$ since schools are considered as objects in the conventional model, students are often assumed to be acceptable at any school. Hence, it is worthwhile to point out that all of our results except Lemma 1 would carry over to the smaller domain of acceptant priorities where any student is acceptable to any school. Since Lemma 1 does not hold (since dropping strategies involve reporting students unacceptable), we do not know whether the large market result would still be true in that case.

\footnotetext{
${ }^{13} \mathrm{~A}$ student $i$ is unacceptable to school $c$ if $\emptyset \succ_{c} i$.

${ }^{14}$ As certain schools can determine their priorities at Boston and New York City school districts, they can declare students unacceptable. Besides, in some school districts, students might not be acceptable due to the living outside of the districts or discipline problems as well. For instance, not all school districts in Massachusetts accept students from outside of their districts.
} 


\section{Appendix}

A mechanism $\psi$ is group strategy-proof if there are no group of students $A \subseteq S$ and a false preference profile for them $P_{A}^{\prime}$ such that $\psi_{i}\left(P_{A}^{\prime}, P_{-A}\right) R_{i} \psi_{i}(P)^{15}$ for all $i \in A$, with holding strictly for at least one student in $A$.

Mechanism $\psi$ is efficient if there is no matching $\mu$ such that $\mu_{i} R_{i} \psi_{i}(P)$ for all $i \in S$, with holding strictly for at least one student.

The following definitions are due to Kojima and Manea (2010).

A preference profile $R_{i}^{\prime}$ is individually rational monotonic transformation of $R_{i}$ at $c \in$ $C \cup\{\emptyset\}\left(R_{i}^{\prime}\right.$ i.r.m.t $R_{i}$ at $\left.c\right)$ if $c^{\prime} R_{i}^{\prime} c$ and $c^{\prime} R_{i}^{\prime} \emptyset \Rightarrow c^{\prime} R_{i} c$ for all $c^{\prime} \in C$; and $R^{\prime}$ i.r.m.t $R$ at a matching $\mu$ if $R_{i}^{\prime}$ i.r.m.t $R_{i}$ at $\mu_{i}$ for all $i \in S$.

A mechanism $\psi$ satisfies individually rational monotonicity if $R^{\prime}$ i.r.m.t $R$ at $\psi(R)$, then $\psi_{i}\left(R^{\prime}\right) R_{i}^{\prime} \psi_{i}(R)$ for all $i \in S$.

Proof of Theorem 1. We prove by contradiction. Let us assume that $\psi^{S}$ is not weakly fictitious student-proof under acyclicity. It implies that there exist a school $c$, matching problem instances $(S, C, P, \succ, q)$ and $\left(S^{\prime}, C, P^{\prime}, \succ^{\prime}, q\right)$ such that $(i)\left(\succ^{\prime}, q\right)$ is acyclic, and (ii) the following conditions satisfy:

(i) $S \subset S^{\prime}$

(ii) for all $i, j \in S$ and $c \in C, i \succ_{c} j$ if and only if $i \succ_{c}^{\prime} j$,

(iii) $P_{i}^{\prime}=P_{i}$ for all $i \in S$,

(iv) for all $i \in S^{\prime} \backslash S$, either $\psi_{i}^{S}\left(S^{\prime}, P^{\prime}, \succ^{\prime}\right)=c$ or $\psi_{i}^{S}\left(S^{\prime}, P^{\prime}, \succ^{\prime}\right)=\emptyset$,

(v) $\psi_{c}^{S}\left(S^{\prime}, P^{\prime}, \succ^{\prime}\right) \cap S \succ_{c} \psi_{c}^{S}(S, P, \succ)$.

Now, consider the following preference profile for students in $S^{\prime}$ :

$$
P_{i}^{\prime \prime}= \begin{cases}\psi_{i}^{S}(S, P, \succ), \psi_{i}^{S}\left(S^{\prime}, P^{\prime}, \succ^{\prime}\right), \emptyset & \text { if } i \in S \\ \psi_{i}^{S}\left(S^{\prime}, P^{\prime}, \succ^{\prime}\right), \emptyset & \text { otherwise }\end{cases}
$$

\footnotetext{
${ }^{15} P_{A}^{\prime}$ and $P_{-A}$ stand for the preference profile of group of student $A$ and that of the rest of the students, respectively.
} 
Let $P^{\prime \prime}=\left(P_{i}^{\prime \prime}\right)_{i \in S^{\prime}}$. Then, we claim that $\psi_{i}^{S}\left(S^{\prime}, P^{\prime \prime}, \succ^{\prime}\right) R_{i}^{\prime} \psi_{i}^{S}\left(S^{\prime}, P^{\prime}, \succ^{\prime}\right)$ for all $i \in S^{\prime}$, with holding strictly for some $j \in S$. Once we prove this claim, proof will be finished since it would contradict the group strategy-proofness of $\psi^{S}$ under acyclic priority structures (Ergin $(2002))$.

For ease of notation, let $\mu^{0}=\psi^{S}(S, P, \succ), \mu^{1}=\psi^{S}\left(S^{\prime}, P^{\prime}, \succ^{\prime}\right)$, and $\mu^{2}=\psi^{S}\left(S^{\prime}, P^{\prime \prime}, \succ^{\prime}\right)$.

First, from the well-known comparative statistics result (Gale and Sotomayor (1985)), $\mu_{i}^{0} R_{i} \mu_{i}^{1}$ for all $i \in S$, which means $\mu_{i}^{0} R_{i}^{\prime} \mu_{i}^{1}$ (since $R_{i}^{\prime}=R_{i}$ for all $i \in S$ ). On the other hand, by the definition of $P^{\prime \prime}, \mu_{i}^{0} R_{i}^{\prime \prime} \emptyset$ for all $i \in S$. Therefore, $R^{\prime \prime}$ i.r.m.t $R^{\prime}$ at $\mu^{1}$. From Kojima and Manea (2010), we know that $\psi^{S}$ satisfies individually rational monotonicity which implies that $\mu_{i}^{2} R_{i}^{\prime \prime} \mu_{i}^{1}$ for all $i \in S^{\prime}$. Then, by the definition of $P^{\prime \prime}$, we have $\mu_{i}^{2} R_{i}^{\prime} \mu_{i}^{1}$ for all $i \in S^{\prime}$.

Next, by our starting supposition, we have $\mu_{c}^{1} \cap S \succ_{c} \mu_{c}^{0}$. This implies that there exists a student $i \in S$ such that $(i) i \in \mu_{c}^{1} \backslash \mu_{c}^{0}$ and $(i i) i \succ_{c} j$ for some $j \in \mu_{c}^{0}(j$ might be $\emptyset$, which means that school $c$ has empty seat under $\mu_{c}^{0}$ and prefers student $i$ to $\emptyset$ ). This along with the stability of $\mu^{0}$ implies that $\mu_{i}^{0} P_{i} \mu_{i}^{1}$, which means $\mu_{i}^{0} P_{i}^{\prime} \mu_{i}^{1}$.

Now, we claim that $\mu_{i}^{2} P_{i}^{\prime} \mu_{i}^{1}$. We prove by contradiction: let us assume that $\mu_{i}^{1}=\mu_{i}^{2}=c .{ }^{16}$ Since $\mu_{i}^{0} P_{i}^{\prime} \mu_{i}^{1}$, it is also true that $\mu_{i}^{0} P_{i}^{\prime} \mu_{i}^{2}$. Then, given $\mu_{k}^{2} \in\{c, \emptyset\}$ for all $k \in S^{\prime} \backslash S$ (by Condition $(i v)$ ), it implies that there exists a student $j \in S, j \neq i$, such that $\mu_{i}^{0}=\mu_{j}^{2}$, $\mu_{j}^{0} \neq \mu_{j}^{2}$ (these are due to the facts that school $\mu_{i}^{0}$ has no excess capacity under $\mu^{2}$ (otherwise it can not be stable) and $\mu_{i}^{0} \neq \mu_{i}^{2}$ ), and $j \succ_{\mu_{i}^{0}} i$ (due to the stability of $\mu^{2}$ ). Moreover, since $\mu^{0}$ is stable in the problem $(S, C, P, \succ, q)$, we have $\mu_{j}^{0} P_{j} \mu_{j}^{2}$, which means $\mu_{j}^{0} P_{j}^{\prime} \mu_{j}^{2}$.

Now, let students $i, j$ and school $\mu_{i}^{0}$ point to schools $\mu_{i}^{0}, \mu_{j}^{0}$ and student $j$, respectively, that is, we consider the following sequence:

$$
i \rightarrow \mu_{i}^{0} \rightarrow j \rightarrow \mu_{j}^{0}
$$

Then, we have the following two cases:

\footnotetext{
${ }^{16}$ Recall that we already proved $\mu_{i}^{2} R_{i}^{\prime} \mu_{i}^{1}$.
} 
Case 1. If $\mu_{j}^{0}=\mu_{i}^{2}$, then let $\mu_{j}^{0}$ point to student $i$. We, hence, end up with the following:

$$
i \rightarrow \mu_{i}^{0} \rightarrow j \rightarrow \mu_{j}^{0} \rightarrow i
$$

The above situation is called "improvement cycle" in the literature in the sense that there is a room for improving efficiency by letting students $i, j$ trade their respective assignments under $\mu^{2}$. Let us denote the matching obtained by implementing this trade while keeping the other students' assignments unchanged by $\tilde{\mu}$. Then, $\tilde{\mu}$ Pareto dominates $\mu^{2}$ with respect to preference profile $P^{\prime}$. On the other hand, since $\mu_{i}^{2} R_{i}^{\prime} \mu_{i}^{1}$ for all $i \in S^{\prime}, \tilde{\mu}$ is also Pareto superior to $\mu^{1}$ in the problem $\left(S^{\prime}, C, P^{\prime}, \succ^{\prime}, q\right)$. This, however, contradicts the fact that $\psi^{S}$ is efficient under acyclic priority structures (Ergin (2002)).

\section{Case 2.}

Step 1. If $\mu_{j}^{0} \neq \mu_{i}^{2}=c$, then, since $\mu_{j}^{0} P_{j}^{\prime} \mu_{j}^{2}$, by the same reasoning as before, there exists a student $k \in S$ different than both $i$ and $j$ such that $\mu_{j}^{0}=\mu_{k}^{2}, \mu_{k}^{0} \neq \mu_{k}^{2}$, and $k \succ_{\mu_{j}^{0}} j$. Moreover, since $\mu^{0}$ is stable in the problem $(S, C, P, \succ, q)$, we have $\mu_{k}^{0} P_{k} \mu_{k}^{2}$, which means that $\mu_{k}^{0} P_{k}^{\prime} \mu_{k}^{2}$.

Now, let school $\mu_{j}^{0}$ and student $k$ point to student $k$ and school $\mu_{k}^{0}$, respectively. Hence, we end up with the following sequence:

$$
i \rightarrow \mu_{i}^{0} \rightarrow j \rightarrow \mu_{j}^{0} \rightarrow k \rightarrow \mu_{k}^{0}
$$

Step 2. Similar to Case 1, if there exists a student in the above sequence who is matched with $\mu_{k}^{0}$ under $\mu^{2}$, let $\mu_{k}^{0}$ point to that student. Let us say this student is $j$, then we have the following:

$$
i \rightarrow \mu_{i}^{0} \rightarrow j \rightarrow \mu_{j}^{0} \rightarrow k \rightarrow \mu_{k}^{0} \rightarrow j
$$

In this case, we also end up with the improvement cycle consisting of students $j, k$ and schools $\mu_{j}^{0}, \mu_{k}^{0}$. If we denote the matching obtained by implementing this cycle while keeping 
the other students' assignments unchanged by $\hat{\mu}$, then $\hat{\mu}$ Pareto dominates $\mu^{2}$ with respect to preference profile $P^{\prime}$, which implies that it also dominates $\mu^{1}$ in the problem $\left(S^{\prime}, C, P^{\prime}, \succ^{\prime}, q\right)$. This, however, contradicts $\psi^{S}$ being efficient under acyclic priority structures.

Step 3. If there exists no student in the sequence (3) who is matched with $\mu_{k}^{0}$ under $\mu^{2}$, then this implies that $\mu_{k}^{0} \neq c$ (Since, otherwise, $\mu_{k}^{0}$ would point to student $i$, who is matched with school $c$ under $\mu^{2}$ by our supposition). Then, by the same reasoning as before, there exists a student $h \in S$ different than $i, j, k$ such that $\mu_{k}^{0}=\mu_{h}^{2}, \mu_{h}^{0} \neq \mu_{h}^{2}$, and $h \succ_{\mu_{k}^{0}} k$. Moreover, since $\mu^{0}$ is stable in the problem $(S, C, P, \succ, q), \mu_{h}^{0} P_{h} \mu_{h}^{2}$, which means $\mu_{h}^{0} P_{h}^{\prime} \mu_{h}^{2}$. Now, let school $\mu_{k}^{0}$ and student $h$ point to student $h$ and school $\mu_{h}^{0}$, respectively. We, therefore, end up with the following sequence:

$$
i \rightarrow \mu_{i}^{0} \rightarrow j \rightarrow \mu_{j}^{0} \rightarrow k \rightarrow \mu_{k}^{0} \rightarrow h \rightarrow \mu_{h}^{0}
$$

Then, if we continue in the same way as before, since everything is finite, we will end up with an improvement cycle. If we denote the matching obtained by implementing that cycle while keeping the other students' assignments unchanged by $\mu^{\prime}$, then $\mu^{\prime}$ Pareto dominates $\mu^{2}$ with respect to $P^{\prime}$, which implies that it is also Pareto superior to $\mu^{1}$ in the problem $\left(S^{\prime}, C, P^{\prime}, \succ^{\prime}, q\right)$. This, however, contradicts the fact that $\psi^{S}$ is efficient under acyclic priority structures.

Therefore, we show that $\mu_{i}^{2} P_{i}^{\prime} \mu_{i}^{1}$ while $\mu_{j}^{2} R_{j}^{\prime} \mu_{j}^{1}$ for all $j \in S^{\prime}$. This, however, contradicts $\psi^{S}$ being group strategy-proof under acyclic priority structures (Ergin (2002)), completing the proof of the weak fictitious student-proofness of $\psi^{S}$ under acyclicity.

Now, for the lack of weak fictitious student-proofness under acyclicity of $\psi^{C}$, we can consider the problem instance given in the proof of Proposition 2. The priority structure given there for $\psi^{C},\left(\succ^{\prime}, q_{a}=2, q_{b}=1\right)$ is acyclic, yet, $\psi^{C}$ is still strongly manipulable. Hence, $\psi^{C}$ is not weakly fictitious student-proof under acyclicity. 
Proof of Theorem 2. Assume for a contradiction that $\psi^{C}$ is not weakly fictitious studentproof under essential homogeneity. This means that there exist a school $c^{\prime}$ and matching problem instances $(S, C, P, \succ, q)$ and $\left(S^{\prime}, C, P^{\prime}, \succ^{\prime}, q\right)$ such that $\left(\succ^{\prime}, q\right)$ is essentially homogeneous and the following conditions satisfy:

(i) $S \subset S^{\prime}$

(ii) for all $i, j \in S$ and $c \in C, i \succ_{c} j$ if and only if $i \succ_{c}^{\prime} j$,

(iii) $P_{i}^{\prime}=P_{i}$ for all $i \in S$,

(iv) for all $i \in S^{\prime} \backslash S$, either $\psi_{i}^{C}\left(S^{\prime}, P^{\prime}, \succ^{\prime}\right)=c^{\prime}$ or $\psi_{i}^{C}\left(S^{\prime}, P^{\prime}, \succ^{\prime}\right)=\emptyset$,

(v) $\psi_{c^{\prime}}^{C}\left(S^{\prime}, P^{\prime}, \succ^{\prime}\right) \cap S \succ_{c^{\prime}} \psi_{c^{\prime}}^{C}(S, P, \succ)$.

Condition (iv) above does not imply that no school in $C \backslash\left\{c^{\prime}\right\}$ makes offers to fictitious students in the course of the school-proposing deferred acceptance procedure. Schools in $C \backslash\left\{c^{\prime}\right\}$ can make offer like school $c^{\prime}$, yet, that condition says that their offers are rejected in some step of the procedure. Moreover, it is easy to observe that any fictitious student in $S^{\prime} \backslash S$ who is unassigned has no effect on the outcome as it implies that either no school makes offer to him or he declares all schools from which he receives offer unacceptable. Therefore, without loss of generality, we assume that any fictitious student $i \in S^{\prime} \backslash \mathrm{S}$ is matched with school $c^{\prime}$ under $\psi^{C}\left(S^{\prime}, P^{\prime}, \succ^{\prime}\right)$. For ease of notation, hereafter, we write $\mu$ and $\mu^{\prime}$ for the outcomes $\psi^{C}(S, P, \succ)$ and $\psi^{C}\left(S^{\prime}, P^{\prime}, \succ^{\prime}\right)$, respectively.

As $S \subset S^{\prime}$ and all fictitious students are matched with school $c$, by the well-known comparative statistics (Gale and Sotomayor (1985)), either $\mu_{c}^{\prime} \succ_{c}^{\prime} \mu_{c}$ or $\mu_{c}^{\prime}=\mu_{c}$ for any school $c \in C \backslash\left\{c^{\prime}\right\}$, with former holding for at least one school in $C \backslash\left\{c^{\prime}\right\}$. Moreover, by our supposition, we have $\mu_{c^{\prime}}^{\prime} \cap S \succ_{c^{\prime}}^{\prime} \mu_{c^{\prime}}$ (note that $\succ_{c}^{\prime}$ over $S$ is the same as $\succ_{c}$ by our supposition).

Let $C^{\prime}=\left\{c \in C: \mu_{c}^{\prime} \neq \mu_{c}\right\}$. Let us pick a school $c \in C^{\prime}$. By our observation above, there exists a student $i \in S$ such that $i \in \mu_{c}^{\prime} \backslash \mu_{c}$ and $i \succ_{c}^{\prime} j$ for some $j \in \mu_{c}$. On the other hand, as $\mu$ is stable, $\mu_{i} \neq \emptyset$. Let $\mu_{i}=\tilde{c}$ and $\tilde{c} \in C^{\prime}$. Due to the stability of $\mu$ and $i \succ_{c}^{\prime} j$, we have $\tilde{c} P_{i} \mu_{i}^{\prime}=c$. Hence, this along with the stability of $\mu^{\prime}$ implies that there exists a 
student $k \in S$ such that $k \in \mu_{\tilde{c}}^{\prime} \backslash \mu_{\tilde{c}}$ and $k \succ_{\tilde{c}}^{\prime} i$. By the same reasoning as above, $\mu_{k} \neq \emptyset$ and let $\mu_{k}=\bar{c}$. That is, we have the following:

$$
k \succ_{\tilde{c}}^{\prime} i \succ_{c}^{\prime} j \text { with } \mu_{i}=\tilde{c} \text { and } \mu_{k}=\bar{c}
$$

If we continue in the same way as above, as everything is finite, we would end up with a set of schools $\left(c_{k}\right)_{k=1}^{n}$ where each of them in $C^{\prime}$ and a set of non-fictitious students $\left(i_{k}\right)_{k=1}^{n+1}$ such that

(i) $i_{1} \succ_{c_{1}}^{\prime} i_{2} \succ_{c_{2}}^{\prime} i_{3}, \ldots, i_{n} \succ_{c_{n}}^{\prime} i_{n+1}=i_{1}$, and

(ii) $\mu_{i_{k+1}}=c_{k}$ and $\mu_{i_{k}}^{\prime}=c_{k}$ for each $k=1, \ldots, n$.

Now, let us consider the assignments of above schools in cycle $(i)$ under matching $\mu^{\prime}$. For each $c_{k}, \mu_{c_{k}}^{\prime} \backslash\left\{i_{k}\right\} \subseteq S^{\prime} \backslash\left\{i_{k}, i_{k+1}\right\},\left|\mu_{c_{k}}^{\prime}\right|=q_{c_{k}}-1$, and $i \succ_{c_{k}}^{\prime} i_{k+1}$ for any $i \in \mu_{c_{k}}^{\prime}$. This is due to the facts that $c_{k}=\mu_{i_{k+1}} P_{i_{k+1}} \mu_{i_{k+1}}^{\prime}=c_{k+1}$ and $\mu^{\prime}$ being stable. Let us write $S_{i_{k+1}}=\mu_{c_{k}}^{\prime}$ for $k=1, . ., n$.

Now, we will create a cycle from $(i)$ consisting of only two schools and two students. First, if $n=2$ in the above construction, then we are done. Let us assume that $n>2$. Then, it implies that $i_{1} \succ_{c_{2}}^{\prime} i_{2}$. As, otherwise, we would have $i_{1} \succ_{c_{1}}^{\prime} i_{2} \succ_{c_{2}}^{\prime} i_{1}$. Now, we can shorten our above cycle by removing school $c_{1}$ and student $i_{2}$. That is, we can consider the following instead of $(i)$ above:

$$
i_{1} \succ_{c_{2}}^{\prime} i_{3} \succ_{c_{3}}^{\prime} i_{4} \ldots, i_{n} \succ_{c_{n}}^{\prime} i_{n+1}=i_{1}
$$

Therefore, we now have a cycle of reduced length by one. Moreover, from above, we know that $S_{i_{3}} \subseteq S^{\prime} \backslash\left\{i_{2}, i_{3}\right\}$ such that $\left|S_{i_{3}}\right|=q_{c_{2}}-1$ and $i \succ_{c_{2}}^{\prime} i_{3}$ for any $i \in S_{i_{3}}$. Moreover, as $i_{1} \notin S_{i_{3}}$ (since $\mu_{i_{1}}^{\prime}=c_{1}$ and $\left.S_{i_{3}}=\mu_{c_{2}}^{\prime}\right), S_{i_{3}} \subseteq S^{\prime} \backslash\left\{i_{1}, i_{3}\right\}$ such that $\left|S_{i_{3}}\right|=q_{c_{2}}-1$ and $i \succ_{c_{2}}^{\prime} i_{3}$ for any $i \in S_{i_{3}}$. We can continue in the same way until we have a cycle consisting of only two schools and two students. Therefore, at the end, we would have two schools $a, b \in C^{\prime}$ and two students $i, j \in S$ such that

$$
i \succ_{a}^{\prime} j \succ_{b}^{\prime} i
$$


Moreover, by the same as above, there exist sets of students $S_{j}, S_{i} \subseteq S^{\prime} \backslash\{i, j\}$ such that $\left|S_{i}\right|=q_{b}-1,\left|S_{j}\right|=q_{a}-1, k \succ_{a}^{\prime} j$ for every $k \in S_{j}$, and $k \succ_{b}^{\prime} i$ for every $k \in S_{i}$. This, however, contradicts the essential homogeneity of $\left(\succ^{\prime}, q\right)$, finishing the proof.

Proof of Theorem 3. We first show that $\psi^{S}$ is at least as manipulable as $\psi^{C}$. To this end, let us assume that the latter is strongly manipulable via creating fictitious students at problem $(S, C, P, \succ, q)$ by school $c$. This means that there exists $\left(S^{\prime}, C, P^{\prime}, \succ^{\prime}, q\right)$ such that the followings hold:

(i) $S \subset S^{\prime}$

(ii) for all $i, j \in S$ and $c \in C, i \succ_{c} j$ if and only if $i \succ_{c}^{\prime} j$,

(iii) $P_{i}^{\prime}=P_{i}$ for all $i \in S$,

(iv) for all $i \in S^{\prime} \backslash S$, either $\psi_{i}^{C}\left(S^{\prime}, P^{\prime}, \succ^{\prime}\right)=c$ or $\psi_{i}^{C}\left(S^{\prime}, P^{\prime}, \succ^{\prime}\right)=\emptyset$,

(v) $\psi_{c}^{C}\left(S^{\prime}, P^{\prime}, \succ^{\prime}\right) \cap S \succ_{c} \psi_{c}^{C}(S, P, \succ)$.

For ease of notation, let $\mu=\psi^{C}(S, P, \succ)$ and $\mu^{\prime}=\psi^{C}\left(S^{\prime}, P^{\prime}, \succ^{\prime}\right)$. We first show that any student $i \in \mu_{c}^{\prime}$ has higher priority than any student $j \in \mu_{c} \backslash \mu_{c}^{\prime}$. For this purpose, first observe that, for any student $j \in \mu_{c} \backslash \mu_{c}^{\prime}, c P_{j} \mu_{j}^{\prime}$ (due to the well-known comparative statistics result of Gale and Sotomayor (1985)). As $\mu^{\prime}$ is stable, it implies that any student $i \in \mu_{c}^{\prime} \backslash \mu_{c}$ has higher priority than anyone else in $\mu_{c}$. Let us write $\underline{s}$ for the student in $\mu_{c}^{\prime} \cap S$ having the lowest priority at school $c$.

Let us consider the priority order $\succ_{c}^{\prime \prime}$ for school $c$ over $S$ under which the relative ordering of students having higher priority than $\underline{s}$ is the same as $\succ_{c}$, and all other students are unacceptable. We write $\succ^{\prime \prime}=\left(\succ_{c}^{\prime \prime}, \succ_{-c}\right)$. We now claim that $\psi_{c}^{C}\left(S, P, \succ^{\prime \prime}\right)=\mu_{c}^{\prime} \cap S$. To this end, let us define matching $\mu^{\prime S}$ as in below:

$$
\mu_{c^{\prime}}^{\prime S}= \begin{cases}\mu_{c^{\prime}}^{\prime} \cap S & \text { If } c^{\prime}=c \\ \mu_{c^{\prime}}^{\prime} & \text { otherwise }\end{cases}
$$

We first need to observe that $\mu^{\prime S}$ is stable at $\left(S, C, P, \succ^{\prime \prime}, q\right)$. As, under $\mu^{\prime S}$, no school other than school $c$ is matched with a fictitious student in $S^{\prime} \backslash S$ and $\mu^{\prime}$ being stable at 
$\left(S^{\prime}, C, P^{\prime}, \succ^{\prime}, q\right)$, there is no blocking pair involving a school in $C \backslash\{c\}$. On the other hand, school $c$ might have excess capacity under $\mu^{\prime S}$ since we exclude its fictitious student assignment under $\mu^{\prime}$. First, consider the students having lower priority than $\underline{s}$. As they are unacceptable under $\succ_{c}^{\prime \prime}$, they do not form a blocking pair with school $c$. On the other hand, for all other students, the relative ordering under $\succ_{c}^{\prime \prime}$ is the same as that of under $\succ_{c}$, hence, $\succ_{c}^{\prime}$. Therefore, as $\mu^{\prime}$ is stable at $\left(S^{\prime}, C, P^{\prime}, \succ^{\prime}, q\right)$, they do not form a blocking pair with school $c$ as well. Hence, $\mu^{\prime S}$ is stable at $\left(S, C, P, \succ^{\prime \prime}, q\right)$. On the other hand, $\psi^{C}\left(S, P, \succ^{\prime \prime}\right)$ is stable at the same problem as well. Moreover, we know that $\left|\mu_{c}^{\prime S}\right|<q_{c}$ (as school $c$ is matched with fictitious students under $\mu^{\prime}$. If it were not matched with fictitious students, then the outcome would not change by creating fictitious students as explained in detail in the above proof). Therefore, by the rural hospital theorem (Roth (1986)), $\psi_{c}^{C}\left(S, P, \succ^{\prime \prime}\right)=\mu_{c}^{\prime S}=\mu_{c}^{\prime} \cap S$.

In what follows, we will first think of a fictitious student manipulation scenario under $\psi^{S}$ and show that the part of the student-optimal stable matching over $S$ at the artificial problem is stable at $\left(S, C, P, \succ^{\prime \prime}, q\right)$. Then, the result will follow from the rural hospital theorem (Roth (1986)).

Now, consider a set of students $S^{\prime \prime}$ such that $S \subset S^{\prime \prime}$ and $\left|S^{\prime \prime} \backslash S\right|=q_{c}$. For the preference of each fictitious student $i \in S^{\prime \prime} \backslash S$, consider $\tilde{P}_{i}: c, \emptyset$. That is, only the school $c$ is acceptable. We write $\tilde{P}=\left(\tilde{P}_{S^{\prime \prime} \backslash S}, P_{S}\right)$. Lastly, for the priority order of school $c$ over $S^{\prime \prime}$, let us enumerate each fictitious student $k \in S^{\prime \prime} \backslash S$ and write $\# k$ for the index of fictitious student $k$. Then, the priority order of school $c$ over $S^{\prime \prime}, \tilde{\succ}_{c}$, is defined as follows:

$$
\text { For any } i \in S^{\prime \prime} \backslash S \text { and } j \in\left\{k \in S: \underline{s} \succ_{c} k\right\}, i \check{\succ}_{c} k \text {; }
$$

For any $i \in S^{\prime \prime} \backslash S$ and $j \in\left\{k \in S: k \succ_{c} \underline{s}\right\} \cup\{\underline{s}\}, j \tilde{\succ}_{c} i$.

For any $i, j \in S^{\prime \prime} \backslash S, i \tilde{\succ}_{c} j$ iff $\# i>\# j$.

The priority orders of schools $c^{\prime} \in C \backslash\{c\}$ over $S^{\prime \prime}, \tilde{\succ}_{c^{\prime}}$, can be anything as long as the relative ordering over $S$ is preserved by our supposition. Now, let us consider the artificial problem $\left(S^{\prime \prime}, C, \tilde{P}, \tilde{\succ}, q\right)$. Let $\tilde{\mu}=\psi^{S}\left(S^{\prime \prime}, C, \tilde{P}, \tilde{\succ}, q\right)$. As only acceptable school for fictitious 
students in $S^{\prime \prime} \backslash S$ is school $c$, they can matched with only school $c$ at $\tilde{\mu}$. Now, consider the following matching $\tilde{\mu}^{S}$ :

$$
\tilde{\mu}_{c^{\prime}}^{S}= \begin{cases}\tilde{\mu}_{c^{\prime}} \cap S & \text { If } c^{\prime}=c \\ \tilde{\mu} & \text { otherwise }\end{cases}
$$

We now claim that $\tilde{\mu}^{S}$ is stable at $\left(S, C, P, \succ^{\prime \prime}, q\right)$. We will follow the same steps as before in showing the stability of $\mu^{\prime S}$. As, under $\tilde{\mu}$, no school other than school $c$ is matched with a fictitious student in $S^{\prime \prime} \backslash S$ and $\tilde{\mu}$ being stable at $\left(S^{\prime \prime}, C, \tilde{P}, \tilde{\succ}, q\right)$, there is no blocking pair involving a school in $C \backslash\{c\}$. On the other hand, school $c$ might have excess capacity under $\tilde{\mu}^{S}$ since we exclude its fictitious student assignment under $\tilde{\mu}$. However, as all students having lower priority than $\underline{s}$ are unacceptable under $\succ_{c}^{\prime \prime}$, they do not form a blocking pair with school $c$. On the other hand, if any student $k \in S$ such that $k \succ_{c}^{\prime \prime} \emptyset$ were to form a blocking pair with school $c$, then $\tilde{\mu}$ could not have been stable at $\left(S^{\prime \prime}, C, \tilde{P}, \tilde{\succ}, q\right)$ as the relative ordering of such students under $\tilde{\succ}_{c}$ is the same as $\succ_{c}^{\prime \prime}$. Therefore, $\tilde{\mu}^{S}$ is stable at $\left(S, C, P, \succ^{\prime \prime}, q\right)$.

Now, we have two stable matchings $\tilde{\mu}^{S}$ and $\psi^{C}\left(S, P, \succ^{\prime \prime}\right)$ at $\left(S, C, P, \succ^{\prime \prime}, q\right)$. Recall that $\psi_{c}^{C}\left(S, P, \succ^{\prime \prime}\right)=\mu_{c}^{\prime} \cap S$. On the other hand, we know that $\left|\mu_{c}^{\prime} \cap S\right|<q_{c}$. Therefore, by the Rural hospital theorem $(\operatorname{Roth}(1986))$, we have $\tilde{\mu}_{c}^{S}=\mu_{c}^{\prime} \cap S$. This means that $\tilde{\mu}_{c} \cap S \succ_{c} \psi^{S}(S, P, \succ)$, hence, $\psi^{S}$ is weakly manipulable via creating fictitious student proof at $(S, C, P, \succ, q)$ as well, showing that $\psi^{S}$ is at least as manipulable as $\psi^{C}$.

For a problem instance at which $\psi^{C}$ is not strongly manipulable via creating fictitious student, yet, $\psi^{S}$ is manipulable, consider a problem consisting of $S=\{i, j\}$ and $C=\{a, b\}$ with $q_{a}=q_{b}=1$. The preference and priority order profiles are as follows:

$$
\begin{aligned}
& P_{i}: a, b, \emptyset ; P_{j}: b, a, \emptyset ; \\
& \succ_{a}: j, i, \emptyset ; \succ_{b}: i, j, \emptyset .
\end{aligned}
$$

Then, $\psi_{a}^{C}(P)=j$ and $\psi_{b}^{C}(P)=i$, hence, schools do not have incentive to manipulate $\psi^{C}$ as they are already matched with their first choices. However, $\psi_{a}^{S}(P)=i$ and $\psi_{b}^{S}(P)=j$. 
Now, let school $b$ create fictitious student $k$ with $P_{k}: a, b, \emptyset$. Assume that the new priority orders of schools are as follows:

$$
\succ_{a}^{\prime}: j, k, i \text { and } \succ_{b}^{\prime}: i, k, j
$$

Let $P^{\prime}=\left(P_{i}, P_{j}, P_{k}\right)$, then $\psi_{b}^{S}\left(S^{\prime}, P^{\prime}, \succ^{\prime}\right)=i$ and $\psi_{a}^{S}\left(S^{\prime}, P^{\prime}, \succ^{\prime}\right)=j$. Hence, school $b$ is better off, showing the manipulability of $\psi^{S}$.

Proof of Lemma 1. Let us consider a problem instance $(S, C, P, \succ, q)$ and stable mechanism $\psi$. Assume that school $c$ can strongly manipulate $\psi$ at the given problem through creating fictitious students. Let $\left(S^{\prime}, C, P^{\prime}, \succ^{\prime}, q\right)$ be the artificial problem including fictitious students created by school $c$. By our supposition, $\psi\left(S^{\prime}, P^{\prime}, \succ^{\prime}\right) \cap S \succ_{c} \psi(S, P, \succ)$. For ease of notation, let $\mu^{\prime}=\psi\left(S^{\prime}, P^{\prime}, \succ^{\prime}\right)$.

Now let us consider the priority order $\succ_{c}^{\prime \prime}$ over $S$ for school $c$ under which the relative ordering over $\mu_{c}^{\prime} \cap S$ is the same as $\succ_{c}$, and any student who is not in $\mu_{c}^{\prime} \cap S$ is unacceptable. We write $\succ^{\prime \prime}=\left(\succ_{c}^{\prime \prime}, \succ_{-c}\right)$. Below, we define a new matching $\mu^{\prime \prime}$ :

$$
\mu_{c^{\prime}}^{\prime \prime}= \begin{cases}\mu_{c}^{\prime} \cap S & \text { If } c^{\prime}=c \\ \mu_{c^{\prime}}^{\prime} & \text { otherwise }\end{cases}
$$

Note that, as school $c$ strongly manipulate $\psi$ by our supposition, $\mu_{c^{\prime}}^{\prime} \subset S$ for any $c^{\prime} \in$ $C \backslash\{c\}$. We now claim that $\mu^{\prime \prime}$ is stable at $\left(S, C, P, \succ^{\prime \prime}, q\right)$. First, there can not be a blocking pair involving school $c^{\prime} \in C \backslash\{c\}$ as, otherwise, such pair would block matching $\mu^{\prime}$ at $\left(S^{\prime}, C, P^{\prime}, \succ^{\prime}, q\right)$, contradicting the stability of $\mu^{\prime}$. On the other hand, school $c$ is not involved in a blocking pair as any student $i \in S \backslash \mu_{c}^{\prime \prime}$ is unacceptable under $\succ_{c}^{\prime \prime}$. Therefore, $\mu^{\prime \prime}$ is stable at $\left(S, C, P, \succ^{\prime \prime}, q\right)$. On the other hand, $\psi\left(S, P, \succ^{\prime \prime}\right)$ is another stable matching. Then, by the Rural hospital theorem (Roth (1984)), we know that $\left|\mu_{c}^{\prime \prime}\right|=\left|\psi_{c}\left(S, P, \succ^{\prime \prime}\right)\right|$. Moreover, as the group of students $\mu_{c}^{\prime \prime}$ is the only acceptable ones under $\succ_{c}^{\prime \prime}$, we have $\mu_{c}^{\prime \prime}=\psi_{c}\left(S, P, \succ^{\prime \prime}\right)=\mu_{c}^{\prime} \cap S$. This shows that $\mu_{c}^{\prime} \cap S$ can be obtained through dropping strategy $\succ_{c}^{\prime \prime}$ as well, which finishes the proof. 


\section{Acknowledgment}

I am grateful to Muriel Niederle, Parag Pathak and especially Fuhito Kojima for their insightful comments and suggestions. I thank Tim Bresnahan.

\section{References}

Abdulkadiroglu, A. (2011): "Generalized Matching for School Choice," mimeo.

Afacan, M. O. (2011): "Application Fee Manipulations in Two-Sided Matching Markets," mimeo.

(2012): "Group Robust Stability in Matching Markets," Games and Economic Behavior, 74(1), 394-398.

Dubins, L. E. And D. A. Freedman (1981): "Machiavelli and the Gale-Shapley Algorithm," American Mathematical Monthly, 88, 485-494.

Ergin, H. I. (2002): "Efficient Resource Allocation on the Basis of Priorities," Econometrica, 88, 485-494.

Gale, D. And L. S. Shapley (1962): "College Admissions and the Stability of Marriage," American Mathematical Monthly, 69, 9-15.

Gale, D. and M. Sotomayor (1985): "Ms. Machiavelli and the Stable Matching Problem," The American Mathematical Monthly, 92(4), 261-268.

Hatfield, J. W., F. Kojima, and Y. Narita (2011): "Promoting School Competition Through School Choice: A Market Design Approach," mimeo. 
Immorlica, N. And M. Mahdian (2005): "Marriage, honesty, and stability," In Proceedings of the Sixteenth Annual ACM-SIAM Symposium on Discrete Algorithms (SODA), $53-62$.

Kesten, O. (2012): "On two kinds of manipulation for school choice problems," Economic Theory, 51(3), 677-693.

Kojima, F. (2011a): "Efficient Resource Allocation under Multi-unit Demand," mimeo. (2011b): "Robust Stability in Matching Markets," Theoretical Economics, 6(2), 257267.

Kojima, F. And M. Manea (2010): "Axioms For Deferred Acceptance," Econometrica, 78(2), 633-653.

Kojima, F. and P. A. Pathak (2009): "Incentives and Stability in Large Two-Sided Matching Markets," American Economic Review, 99(3), 608-627.

Pathak, P. A. And T. Sönmez (2013): "School Admissions Reform in Chicago and England: Comparing Mechanisms by their Vulnerability to Manipulation," American Economic Review, 103(1), 80-106.

Rotн, A. E. (1982): "The Economics of Matching: Stability and Incentives," Mathematics of Operations Research, 7, 617-628.

(1984): "The Evolution of the Labor Market for Medical Interns and Residents: A Case Study in Game Theory," Journal of Political Economy, 92, 991-1016.

_ (1985): "The college admissions problem is not equivalent to the marriage problem," Journal of Economic Theory, 36, 277-288.

(1986): "On the Allocation of Residents to Rural Hospitals: A General Property of Two-Sided Matching Markets," Econometrica, 54, 425-427. 
Roth, A. E. And E. Peranson (1999): "The Redesign of the Matching Market for American Physicians: Some Engineering Aspects of Economic Design," American Economic Review, 89(4), 748-780.

Roth, A. E. And M. O. Sotomayor (1990): Two-Sided Matching: A Study in GameTheoretic Modeling and Analysis, Econometric Society Monographs, Cambridge Univ. Press, Cambridge.

Sönmez, T. (1997): "Manipulation via Capacities in Two-Sided Matching Markets," Journal of Economic Theory, 77(1), 197-204.

_ (1999): "Can Pre-arranged Matches Be Avoided in Two-Sided Matching Markets?" Journal of Economic Theory, 86, 148-156.

Yokoo, M., Y. Sakurai, and S. Matsubara (2004): "The effect of false-name bids in combinatorial auctions: new fraud in internet auctions," Games and Economic Behavior, 46(1), 174-188. 